Sir,

\section{A case of persistent fetal vasculature with atypical presentaion}

Persistent fetal vasculature (PFV) is a pleomorphic disorder of ocular development due to incomplete regression of the fetal hyaloid vasculature, ${ }^{1}$ and its varied clinical presentations may pose a diagnostic challenge.

\section{Case report}

A 5-year-old boy presented with vision 20/20 OD and counting fingers OS. Examination of the left eye revealed posterior lens opacity and a large retrolental pigmented lesion occluding the visual axis (Figure 1a). B-scan ultrasonography was normal.

Lensectomy and/or biopsy of any mass/cyst was planned, and under anesthesia, the lens appeared subluxated nasally with the notched equator visible temporally (Figures $1 \mathrm{~b}$ and $\mathrm{c}$ ). Horizontal cornea diameters were $11 \mathrm{~mm}$ OU, and axial lengths were $22.79 \mathrm{~mm}$ OD and $25.03 \mathrm{~mm}$ OS. Ultrasound biomicroscopy showed a sheet-like structure arising from the ciliary body, which extended retrolentally and encased an area of posterior lentiglobus (Figure 2). Lensectomy, posterior capsulectomy, anterior vitrectomy, and in-the-sulcus intraocular lens implantation was performed, and intraoperatively, a thin non-perfused fibrovascular stalk was seen running from the optic disc to the posterior capsule. Histopathological examination of the capsulectomy specimen was consistent with stretched ciliary processes associated with PFV (Figure 1d). Post operatively, vision improved to 20/150 (plano/ $-2.75 \times 175$ ) at 20 months, despite poor compliance with patching.

\section{Comments}

The atypical appearance of the grossly stretched ciliary processes raised the suspicion of a ciliary mass/cyst, particularly medulloepithelioma in this age group. Although typically white, medulloepitheliomas can be tan-coloured, and localized absence of zonules and resultant lens abnormalities (coloboma, subluxation, cataract) similar to our patient have been reported. ${ }^{2}$

In PFV, lens subluxation and coloboma can be caused by persistent iridohyaloid vessels interfering with zonular development, and/or traction from the hyaloid artery. ${ }^{1}$ Traction is also implicated in the development of lentiglobus in $\mathrm{PFV}^{3}$ although our case was unusual in that lentiglobus was lined entirely by grossly stretched ciliary processes.

Another atypical feature was the normal-sized cornea and long axial length. Myopic PFV is considered to be a variant of the typical microphthalmic form, and has a better prognosis with less risk of posterior involvement and glaucoma. ${ }^{4}$ These milder forms tend to present later, and may be difficult to diagnose, with some diagnosed only during surgery, ${ }^{5}$ such as our case where the thin fibrovascular stalk was missed on B-scan.

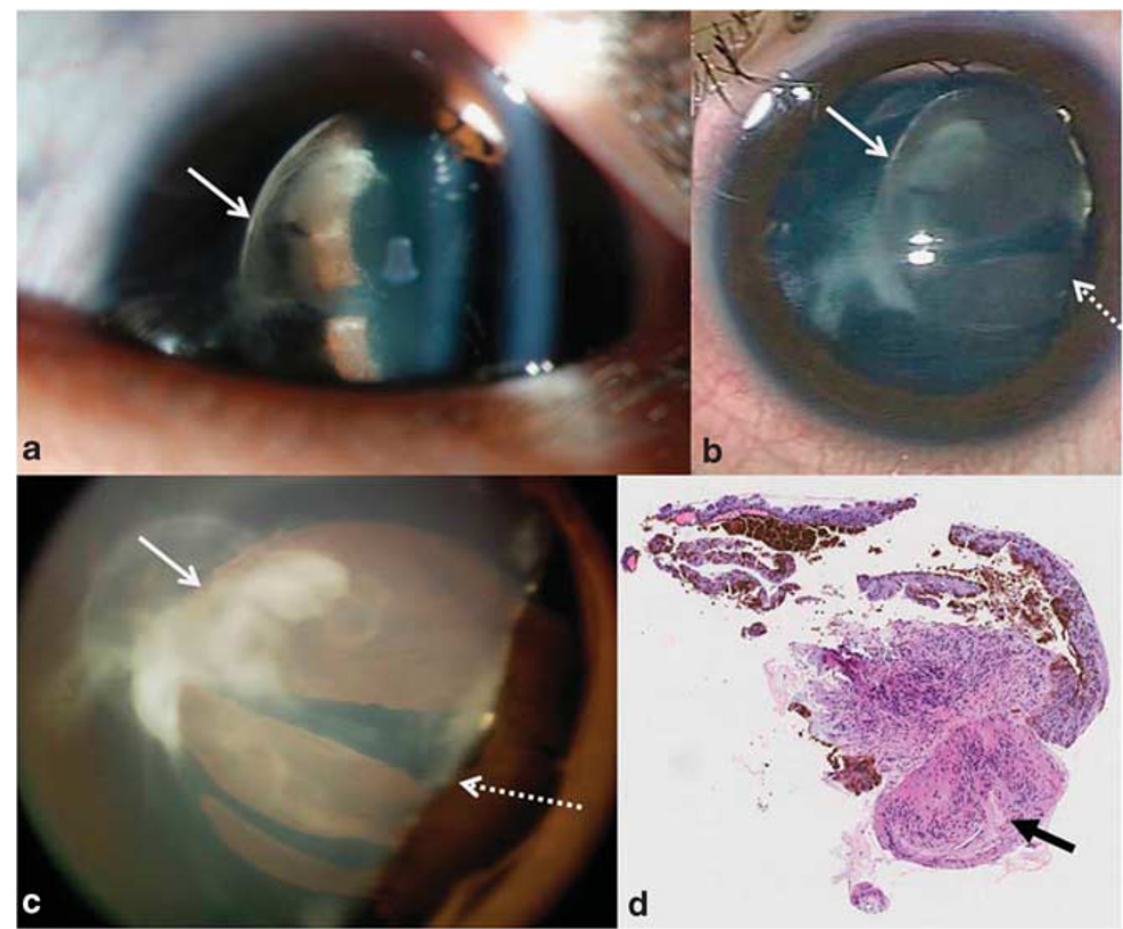

Figure 1 (a) Anterior segment slit lamp photograph, (b) intraoperative image from operating microscope, (c) RetCam image, of the left eye, showing lens opacity, retrolental brown lesion (nasal edge denoted by solid arrow), and colobomatous area of lens from 2 to 5 o'clock (dotted arrow). (d) Histopathological micrograph of the posterior capsulectomy specimen consisting of tissue covered by ciliary body-type epithelium, with underlying smooth muscle cells adjacent to neural-type cells (arrow) (hematoxylin and eosin, original magnification $\times 40$ ). 


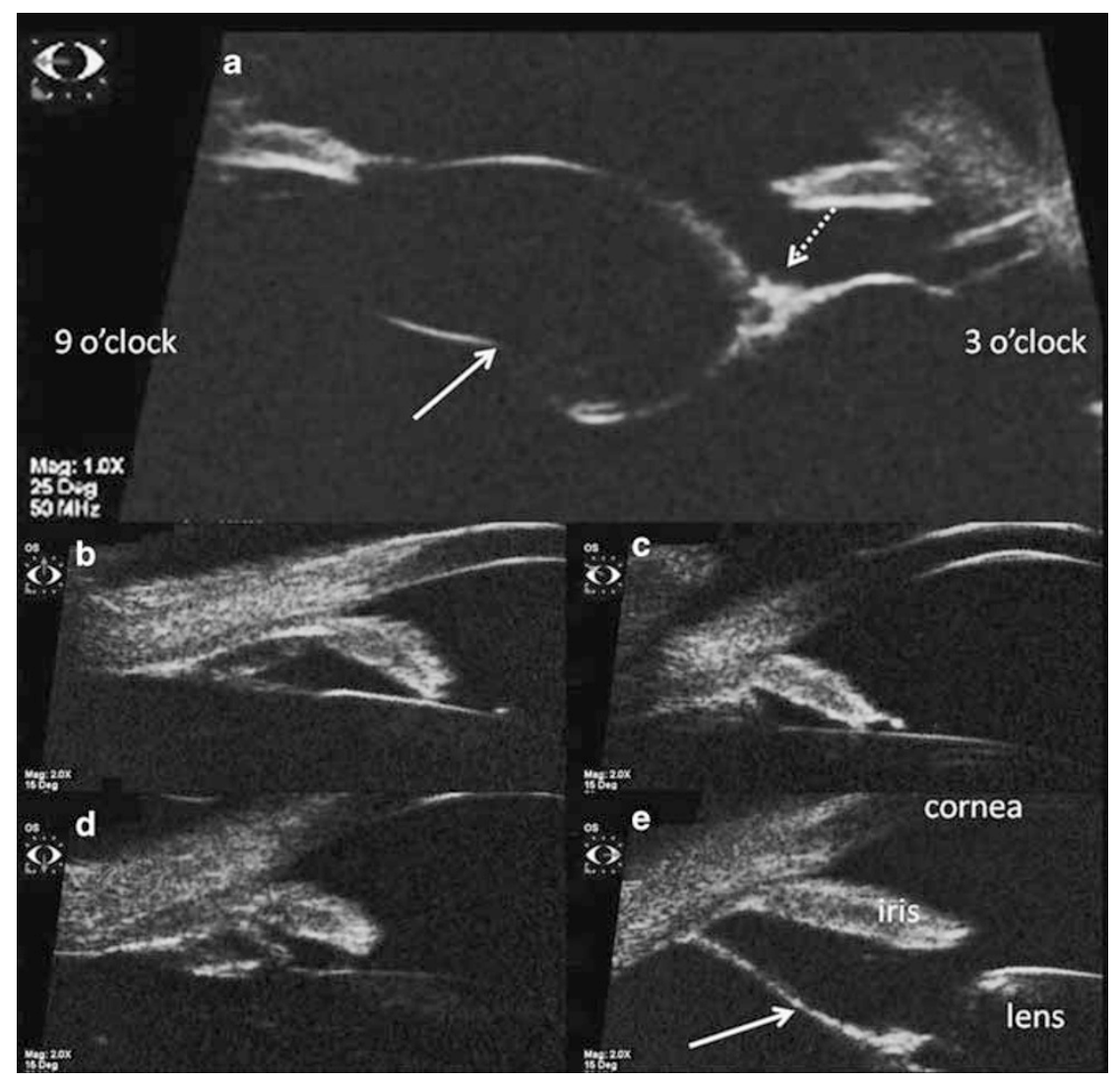

Figure 2 (a) Sulcus-to-sulcus scan of the left eye using ultrasound biomicroscopy (UBM) in the horizontal meridian, showing the extent of the brown lesion, which extended from the ciliary body region at $3 \mathrm{o}^{\prime}$ clock to the back of the lens with posterior bowing. Its nasal extent was denoted by a solid arrow, and the temporal edge of the lens by the dotted arrow. UBM of the left eye, showing normal configuration at superior (b), nasal (c), and inferior (d) aspects. The temporal (e) region showed a flat sheet-like structure (arrow) extending from the ciliary body region to the posterior aspect of the lens.

\section{Conflict of interest}

The authors declare no conflict of interest.

\section{References}

1 Goldberg MF. Persistent fetal vasculature (PFV): an integrated interpretation of signs and symptoms associated with persistent hyperplastic primary vitreous (PHPV). LIV Edward Jackson Memorial Lecture. Am J Ophthalmol 1997; 124: $587-626$.

2 Shields JA, Eagle Jr RC, Shields CL, Potter PD. Congenital neoplasms of the nonpigmented ciliary epithelium (medulloepithelioma). Ophthalmology 1996; 103: 1998-2006

3 Kilty LA, Hiles DA. Unilateral posterior lenticonus with persistent hyaloid artery remnants. Am J Ophthalmol 1993; 116: 104-106.

4 Cheung JC, Summers CG, Young TL. Myopia predicts better outcome in persistent hyperplastic primary vitreous. J Pediatr Ophthalmol Strabismus 1997; 34: 170-176.

5 Müllner-Eidenböck A, Amon M, Hauff W, Klebermass N, Abela C, Moser E. Surgery in unilateral congenital cataract caused by persistent fetal vasculature or minimal fetal vascular remnants: age-related findings and management challenges. J Cataract Refract Surg 2004; 30: 611-619.
KR Darusman', MCD Aquino', TP Thamboo ${ }^{2,3}$ and IB Wong' ${ }^{1,3}$

${ }^{1}$ Department of Ophthalmology, National

University Hospital, Singapore

${ }^{2}$ Department of Pathology, National University

Hospital, Singapore

${ }^{3}$ Yong Loo Lin School of Medicine, National

University Singapore, Singapore

E-mail: inez_by_wong@nuhs.edu.sg

Eye (2011) 25, 1521-1522; doi:10.1038/eye.2011.206; published online 19 August 2011

Sir,

Anterior segment OCT imaging in opaque grafts with secondary glaucoma following tectonic penetrating keratoplasty for perforated corneal ulcers

Penetrating keratoplasty performed for perforated corneal ulcers is associated with a high incidence of secondary glaucoma. ${ }^{1,2}$ Anterior segment optical coherence tomography (ASOCT) provides non-contact, non-invasive, high-resolution, real-time cross-sectional images of the anterior segment of the eye. ${ }^{3,4}$ 